\title{
LANDPOWER AND DUAL CONTAINMENT: RETHINKING AMERICA'S POLICY IN THE GULF
}

Stephen C. Pelletiere

November 1999 